\title{
TLM modeling and system identification of optimized antenna structures
}

\author{
N. Fichtner ${ }^{1}$, U. Siart ${ }^{1}$, Y. Kuznetsov ${ }^{2}$, A. Baev ${ }^{2}$, and P. Russer ${ }^{1}$ \\ ${ }^{1}$ Institute for High Frequency Engineering, Technische Universität München, Arcisstr. 21, 80333 München, Germany \\ ${ }^{2}$ Moscow Aviation Institute (State Technical University) Volokolamskoe sh. 4, A-80, GSP-3, Moscow, 125993, Russia
}

\begin{abstract}
The transmission line matrix (TLM) method in conjunction with the genetic algorithm (GA) is presented for the bandwidth optimization of a low profile patch antenna. The optimization routine is supplemented by a system identification (SI) procedure. By the SI the model parameters of the structure are estimated which is used for a reduction of the total TLM simulation time. The SI utilizes a new stability criterion of the physical poles for the parameter extraction.
\end{abstract}

\section{Introduction}

The modeling of the electromagnetic behavior and the design optimization is an important issue in the development of new systems and services. Fullwave techniques operating in timedomain (TD) as e.g. TLM and FDTD are advantageous for structures where the system response at several frequencies is needed. An efficient global optimization method is available by the genetic algorithm (GA) (Johnson and RahmatSamii, 1997, 1999). The GA is typically used with a methods of moments whereas here it is adopted to the transmission line matrix (TLM) method (Fichtner et al., 2007). Any GA optimization requires a repeated computation of the electromagnetic structure under consideration. In order to lower the computational burden a system identification (SI) methods is applied. On the one side the SI allows a reduction of the total TLM simulation time by estimation of the future signal response. On the other side the SI results in the extraction of the model order and the model's parameters allow for the determination of a lumped element network. The network oriented modeling of passive microwave circuits and antennas provides a compact representation of the electromagnetic

Correspondence to: N. Fichtner (fichtner@tum.de) structures and requires low computational effort and memory capacity.

\section{TLM modeling}

In this paper we investigate methods to reduce the simulation time of the TLM simulation through the model parameter estimation from significant parts of the impulse response using SI methods. As soon as the model parameters can be estimated with sufficient stability, a network oriented equivalent model may be established and the TLM simulation can be truncated at this point. In high- $Q$ resonant structures with long impulse responses this method can save typically up to $90 \%$ of the total TLM simulation time (Kuznetsov et al., 2005). But even in low- $Q$ structures a reduction of $50 \%$ is achievable. Therefore, a SI for the modeling of broadband structures is also advantageous.

The simulation results of the TLM electromagnetic modeling package YATSIM (Yat, 2007) have been used as a basis for the SI procedure. YATSIM provides an open source software package for time-domain electromagnetic full-wave simulation based on the TLM method. Our benchmark example is a low-profile microstrip fed patch antenna that has been optimized for maximum impedance bandwidth using a genetic algorithm (GA). The description of the GA optimization approach is outside the scope of this paper, see e.g. Johnson and Rahmat-Samii $(1997,1999)$ and Fichtner et al. (2007). In the following we compare the SI results for the initial (non-optimized) antenna to the results for the GA optimized antenna. The CAD antenna model of the antenna is shown in Fig. 1. The antenna is designed to have its first resonance mode $T M_{100}$ at $6 \mathrm{GHz}$ and the second resonance mode $T M_{010}$ at $12 \mathrm{GHz}$ (for patch antenna in the xy-plane). The green bridge-like objects in Fig. 1 do not correspond to physical objects but indicate the locations of probes for Eand $\mathrm{H}$-fields, where the time domain impulse response has

Published by Copernicus Publications on behalf of the URSI Landesausschuss in der Bundesrepublik Deutschland e.V. 


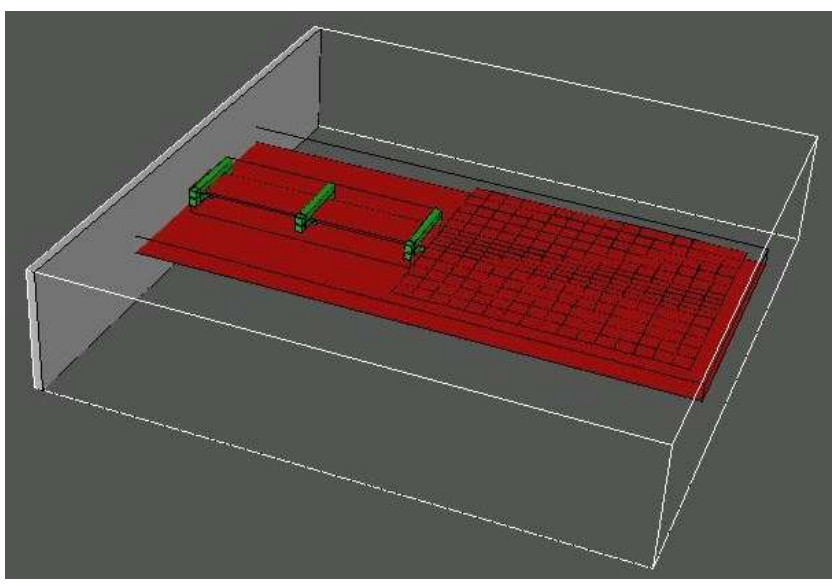

Fig. 1. CAD rendered view of the simulated and optimized patch antenna.

been computed with 10000 time steps with the increment $\Delta t=8.3 \times 10^{-13} \mathrm{~s}$.

\section{System identification}

The SI method applied in this contribution is based on a system model deduced from the singularity expansion method (SEM) (Baum et al., 1991; Heyman and Felsen, 1985). Therein a scattering process in TD is subdivided into the entire part and the pole part. The entire part is responsible for the high frequency processes inside the EM structure concerning reflections, delays and other transformations of the initial wave front. The pole part describes the low frequency processes given by the quasi static exchange of the electric and magnetic energy stored at different locations in the microwave circuit. For a given input signal $a(t)$ we compute the response signal $b(t)$ from the convolution

$b(t)=a(t) \times\left[h_{\mathrm{POLE}}(t)+h_{\mathrm{ENT}}(t)\right]$

where

$h_{\mathrm{POLE}}(t)=\sum_{i=1}^{P} C_{i} e^{-p_{i} t}$

$h_{\mathrm{ENT}}(t)=\sum_{k=1}^{K} D_{K} a\left(t-T_{k}\right)$

are the impulse responses for the pole and entire parts, respectively. The estimation of $D_{k}, T_{k}$ and $K$ in Eq. (3) is not a part of this contribution because the early time part has short duration and will not be approximated by a system model. The residues $C_{i}$ and the poles $p_{i}$ in Eq. (2) characterize the late time response of the scattered signal and are subject to the SI.

In conventional parameter estimation methods such as the Matrix Pencil method (MPM) published by Hua and Sarkar

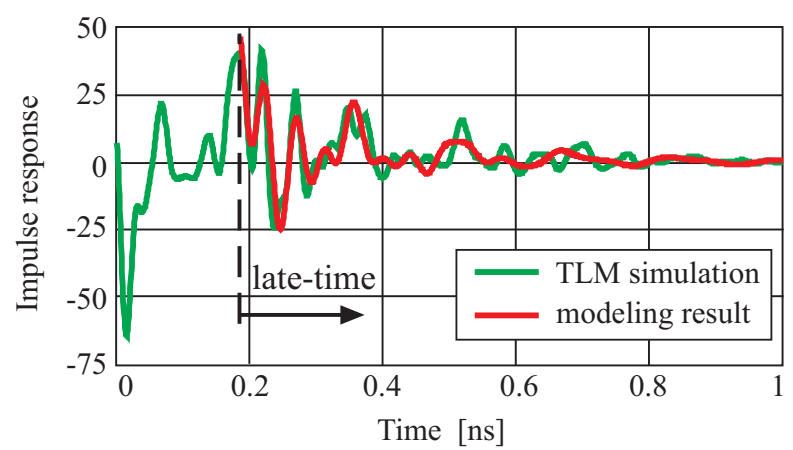

(a) Non-optimized antenna

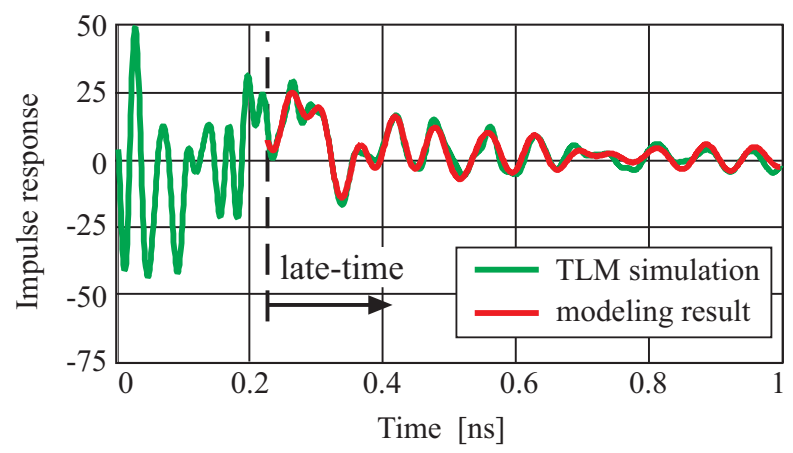

(b) Optimized antenna

Fig. 2. Patch antenna impulse response: TLM simulated and reconstructed by system identification.

(1990) the extracted poles can be categorized into physical and unphysical poles. The first are structure dependent whereas the latter only increase the quality of the approximation. From the SEM we know that the late time response of a back-scattered signal is given by structure dependent resonances. Thus a parameter estimation with a constant observation time window at different times in the late time part should result in constant pole positions for the physical poles. In order to classify the poles we applied the MPM for a constant observation window at different times in the response signal $b(t)$. Two effects were uncovered: Firstly one part of the obtained poles have positions in the complex plane which are independent from the actual observation time. Secondly there exists a minimal time border below which none of the poles has a constant position. The poles independent of the observation times can be considered as physical poles and the remaining poles as unphysical (numerical) poles.

The number of physical poles $P_{0}$ can also be obtained from the proposed SI method. We consider the case with $P>P_{0}$ and compute the pole locations at two different observation times. By this way we obtain two sets of poles $\mathbf{P}_{1}=\left\{p_{1}^{(1)}, \ldots, p_{P}^{(1)}\right\}$ and $\mathbf{P}_{2}=\left\{p_{1}^{(2)}, \ldots, p_{P}^{(2)}\right\}$. Next we 


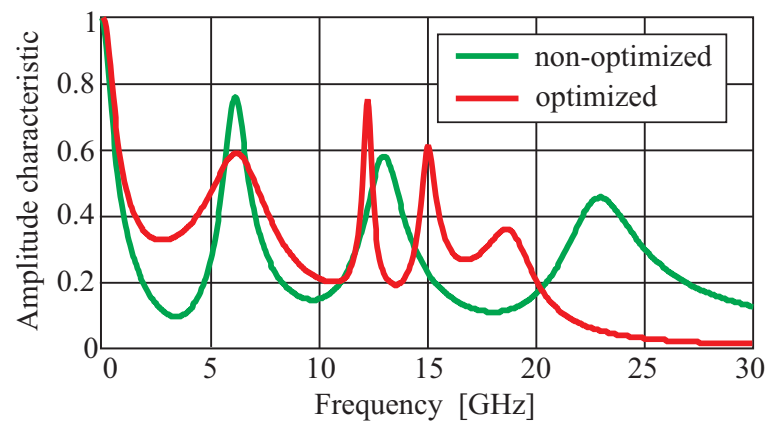

Fig. 3. Modeled patch antenna amplitude spectral response.

compute the Euclidian metric of the two pole sets from

$\Delta P=\left\|\boldsymbol{P}_{1}-\boldsymbol{P}_{2}\right\|=\sqrt{\sum_{n=1}^{P}\left|p_{n}{ }^{(1)}-p_{n}{ }^{(2)}\right|^{2}}$.

Note that in the metric (4) for a pole in set $1 p_{n}^{(1)}$ the closest pole of set 2 must be chosen in order to minimize $\Delta P$. However, the physical poles have almost constant position, i.e. $p_{n}^{(1)} \approx p_{n}^{(2)}$, whereas the unphysical poles deviate considerably and therefore increase $\Delta P$. The metric $\Delta P$ mainly depends on the number of unphysical poles presented in the expansion (2). Choosing a smaller pole number $P$ decreases the metric because some of the unphysical poles will vanish. This works down to $P=P_{0}$. For smaller vales $P<P_{0}$ the physical poles will start to change their position for different observation times which increases the metric $\Delta P$. Thus the number of physical poles $P_{0}$ is found by varying $P$ and looking for the minimum value of $\Delta P$.

\section{Modeling results}

The impulse responses of the non-optimized (initial) antenna and the bandwidth optimized patch antenna described in Sect. 2 have been computed by the proposed SI method. The results are shown in Fig. 2. Figure 3 shows the corresponding amplitude characteristics. It can be seen that the optimized antenna has additional resonant frequencies. These provide a larger impedance bandwidth compared to the initial antenna with two resonances. It has to be mentioned that the additional resonances may considerably influence the group delay properties. Therefore the impedance bandwidth criterion used here applies well to wideband antennas for several narrowband services. However, antennas optimized in that way may not be suitable for ultrawide band pulse transmission.

The estimated model parameters can be used to establish network oriented equivalent models of the microwave structures by means of network synthesis. As an example the antenna input impedance derived from the all-pole model is shown in Fig. 4.
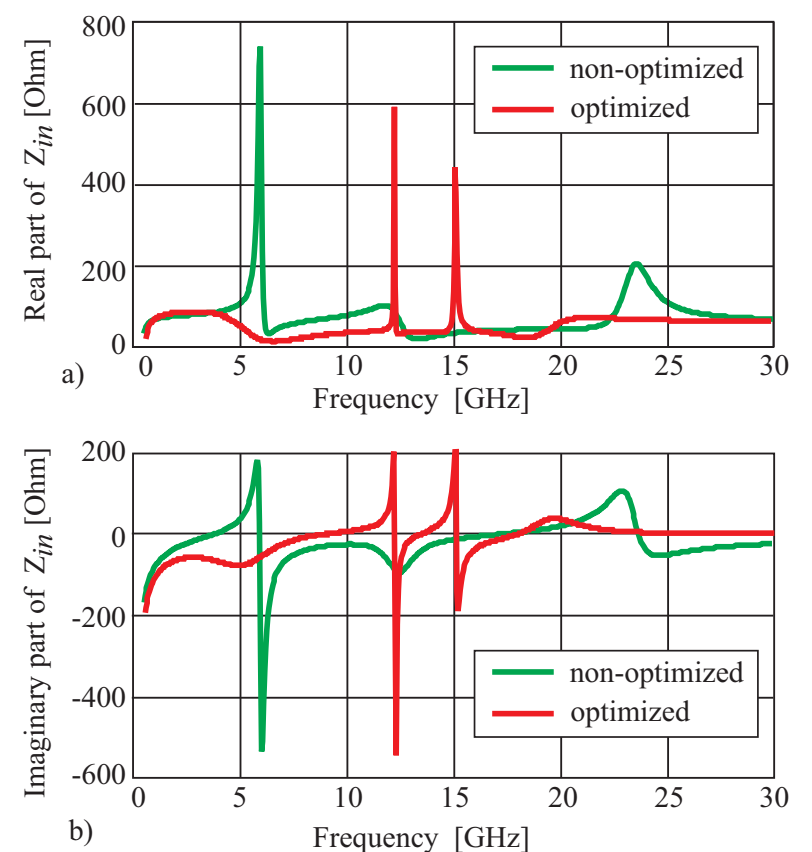

Fig. 4. Modeled patch antenna input impedance.

\section{Conclusions}

The time domain simulation of EM structures is very efficient for broadband structures but may require long simulation time for low loss resonant components. System identification can help to establish network oriented models of the structures from parts of the impulse response thus avoiding the need to simulate the total duration of the scattered signal. A combination of time domain electromagnetic modeling and system identification has therefore the potential to reduce computation time considerably. In this paper system identification has been applied to a bandwidth optimized patch antenna and to a band filter structure with good agreement between full time TLM response and SI based estimation.

Acknowledgements. The authors are indebted to the Deutscher Akademischer Austauschdienst (DAAD) for supporting the scientific exchange between the Moscow Aviation Institute and the Technische Universität München.

\section{References}

Baum, C., Rothwell, E., Chen, K.-M., and Nyquist, D.: The Singularity Expansion Method and Its Application to Target Identification, Proc. IEEE, 79, 1481-1492, 1991.

Fichtner, N., Siart, U., and Russer, P.: Antenna Bandwidth Optimization Using Transmission Line Matrix Modelling and Genetic Algorithms, in: ISSSE Proc. Int. Symp. on Signals, Systems and Electronics, Montreal, Quebec, 2007. 
Heyman, E. and Felsen, L.: A Wavefront Interpretation of the Singularity Expansion Method, IEEE Trans. Antennas Propagat., 33, 706-718, 1985.

Hua, Y. and Sarkar, T.: Matrix pencil method for estimating parameters of exponetially damped/undamped sinusoids in noise, IEEE Trans. Acoustics, Speech and Signal Processing, 38, 814$824,1990$.

Johnson, J. and Rahmat-Samii, Y.: Genetic Algorithms in engineering electromagnetics, IEEE Trans. Antennas Propagat. Magazine, 39, 7-25, 1997.
Johnson, J. and Rahmat-Samii, Y.: Genetic Algorithms and Method of Moments (GA/MOM) for the Design of Integrated Antennas, IEEE Trans. Antennas Propagat., 47, 1606-1614, 1999.

Kuznetsov, Y., Baev, A., Shevgunov, T., Zedler, M., and Russer, P.: Transfer Function Representation of Passive Electromagnetic Structures, in: Proc. MTT-S Int. Microwave Symp., 2005.

Kuznetsov, Y., Baev, A., Shevgunov, T., Lorenz, P., and Russer, P.: Application of the Stability Criterion to the Passive Electromagnetic Structures Modeling, in: Proc. 36th European Microwave Conf., 2006.

YATPAC Homepage: http://www.yatpac.org, 2007. 